\title{
Original Research Article The Role of Morphology in English Vocabulary Teaching
}

\author{
Yajie Jiang* \\ School of foreign language, China West Normal University, Nanchong 637000, China. E-mail: 316027157@qq.com
}

\begin{abstract}
Vocabulary is the most important factor in language composition. Guiding English learners to acquire English vocabulary is an important task. Based on the morphological theory, this research starts from the internal structure of the word and tries to explore the rules of word formation in English vocabulary in order to provide some useful enlightenment for English vocabulary acquisition.
\end{abstract}

Keywords: Morphology; Morpheme; Suffix; Root; English Vocabulary Acquisition

\section{Introduction}

As one of the three elements of language (speech, vocabulary, grammar), vocabulary is the most basic part of language expression. When linguist McCarthy ${ }^{[1]}$ talked about these three relationships, he pointed out: "No matter how well the student learns grammar, no matter how successfully the sounds of L2 (Second Language) are mastered, without words to express a wider range of meanings, communication in an L2 just cannot happen in any meaningful way." Therefore, vocabulary learning is one of the important links in learning a language. Mastering the required vocabulary is the key to understanding or be understood the words in the process of language communication. It turns out that poor vocabulary and vocabulary misuse seriously affect the improvement of learners' levels. Therefore, strengthening the study of vocabulary learning is an important topic for English learners and educators. This article focuses on how to use morphological knowledge to improve vocabulary teaching.

\section{Factors that prevent students from expanding their vocabulary}

For students, not having a large amount of vocabulary is an important factor that hinders students from further improving their English $^{[2]}$. The factors that hinder students from expanding their vocabulary are summarized as follows:

\subsection{Learning English for test purposes}

For English learners in China, many people in English study have only one purpose, that is, pass the exam. Such as college entrance examination, college English test four or six. Many students regard English learning as a burden. Once the learner loses interest in English learning, the effect of vocabulary learning will be greatly reduced.

\subsection{Lack of lexical knowledge in vocabulary teaching}

The traditional way of teaching vocabulary is to let students remember words in isolation, and rarely let students combine language skills with actual needs. For students, it is boring to memorize vocabulary that they are not interested in, and the effect of memory is not ideal. The teacher's teaching stays in the Chinese meaning of the word and the differences between similar words, but rarely considers the composition of the word, morphemes and so on. Students' learning is also to memorize words in isolation from a cognitive perspective or memorize alphabets one by one. Both the teacher's teaching and the student's learning are scientific and systematic, and rarely use morphological knowledge.

\subsection{Passively learning vocabulary}

How does the teacher teach, how do students learn; what does the teacher teach and what the students learn. Students are accustomed to passively accepting the knowledge taught by the teacher, and rarely think and question by themselves.

\section{Morphology}

Morphology is a branch of linguistics that deals with the internal structure of words. To understand the internal structure of a word, you need to know what a morpheme is.

The definition of a morpheme is "a minimal unit of meaning or grammatical function ${ }^{[3]}$." According to different standards, morphemes can be divided into different types ${ }^{[4]}$.

This is an open-access article distributed under the terms of the Creative Commons Attribution Non-Commercial License 


\subsection{Free and bound morphemes}

From the perspective of whether morphemes can express meaning separately, morphemes can be divided into two categories ${ }^{[5]}$. Free morphemes are all roots, which are capable of being used as words word-building elements to form new words. Bound morphemes are bound to other morphemes to form words or to perform a particular grammatical function. Let us take recollection, idealistic and ex-prisoner for example. Each of the three words comprises three morphemes: recollection (re-collect-ion), idealistic (ideal-ist-ic), ex-prisoner (ex-prison-er). Of the nine morphemes, collect, ideal and prison can stand by themselves and thus are free morphemes. All the rest $r e-$, -ion, -ist, ex- and -er are bound as none of them are freestanding units.

\subsection{Derivational and inflectional morphemes}

Morphemes used to derive new words are known as derivational morphemes ${ }^{[6]}$. Because when these morphemes are conjoined, new words are derived. In English, derivatives and compounds are all formed by such morphemes. For example, $a+m o r+a l$, clear + ance, life + like and homo + gen+ eous are results of morphological processes. Inflectional morphemes, in contrast, indicate syntactic relationships between words and thus function as grammatical markers. In English, inflectional morphemes are all suffixes. For instance, the regular plural suffix $-s(-e s)$ is added to nouns such as machines, fridges, desks, radios and potatoes; the same forms call be added to verbs to indicate the simple present for the third person singular such as likes, works and goes.

\subsection{Roots, affixes and stems}

A root is the basic form of a word which cannot be further analyzed without total loss of identity. It is that part of the word that remains when all affixes have been removed. A root can be free or bound. Whether free or bound, it carries the main component of word meaning. Affixes are forms that are attached to bases or stems to change or modify meaning or function. All affixes are bound morphemes because none of them can stand as words on their own. Stem is part of a word-form which remains when inflectional morphemes have been removed. It is used to refer to the form of the word to which both inflectional and derivational morphemes can be added.

\subsection{Composition of words}

Strictly speaking, the composition of words is a variant of expressing the meaning relationship of words. It can be further divided into synthetic and derived. Derivation reflects the relationship between roots and affixes. Derivation can change or maintain the original word class. In English, a word can only have one inflection affix, but can have multiple derivations. For vocabulary learning, the most valuable of morphology is the knowledge of the roots and the composition of words.

\section{The composition and source of English words}

From the perspective of lexical morphology, there are several main ways to develop English vocabulary ${ }^{[7]}$.

\subsection{English loan-words}

In history, Britain was twice invaded and ruled by foreigners on a large scale. During this period, language and culture have been greatly affected. The most influential are Greek and Latin. In addition to this, there are French, German, etc. So there are a lot of foreign words in English, such as alcohol (Arabic), boss (Dutch), croissant (French), lilac (Persian), piano (Italian), pretzel (German), robot (Czech), tycoon (Japanese), yogurt (Turkish). This form of word formation is called borrowing.

\subsection{English conversion words}

In the process of language development, the part of speech of some words will change. For example, when water first appeared, it was a noun. With daily communication, water can be used as the verb watering. It also has the noun paper, which can be used as a verb to paper. This method of word formation is called conversion. Similar words include butter, bottle, vacation, can, etc. It is characterized by the constant form of words and the transformation of part of speech.

\subsection{English abbreviations}

From the morphological structure and morphology of linguistics, English abbreviations can be divided into two categories.

\subsubsection{Acronyms}

If the form is abbreviated according to the initials of the phrase, then this method is called acronym. There are two ways to pronounce such words. One is to pronounce according to the first letter of each word. For example, VOA ['vi: au 'ei] (Voice Of America), VCR ['vi: si`a:] (video cassette recorder), CD [`si: di:] (compact disk). The other one is to read the words in alphabetical order. Such as: NASA [næsə] (National Aeronautics and Space Administration), NATO ['neitəu] (North Atlantic Treaty Organization), UNESCO [ju: neskə] (United Nations Educational, Scientific, and Cultural Organization). Most of the acronyms are organizational names or some technical terms.

\subsubsection{Clipping}

A new word formed by reducing one or more syllable parts of a word is called a clipping ${ }^{[8]}$. The most common types of shortening words are: 1. Shorten the head of the word. Phone =telephone, plane=airplane. 2. Shorten the head and tail of the word. Flu $=$ influenza, tec $=$ detective, fridge $=$ refrigerator. 3 . Reduce the tail. ad $=$ advertisement, pro=professional, Prof $=$ Professor, $\mathrm{Ed}=$ Edward, Sam=Samuel. 4. Eliminate the middle part of the word, take the first and last words of the word. Mr=Mister, $\mathrm{Dr}=$ Doctor, Tom=Thomas. The most commonly used of the four shortened words listed is the third one, followed by the first one, and the second and fourth words are few.

\section{The application of morphology in English vocabulary teaching}

When learners have mastered certain grammar and reading skills, they should systematically learn the knowledge of English 
roots and word formation, which is the best way to expand vocabulary ${ }^{[9]}$. At this stage, they should learn some basic word formation knowledge and master some roots and morphemes. Mastering some of the knowledge of word formation makes it easier to remember and use words, and to make English vocabulary learning interesting.

The main objects of morphological research are the internal structure of words, words and rules of word formation. The research results in this area will directly benefit the vocabulary learning of second language learners. If the rules of word formation can be used by learners, it will certainly change or accelerate the process of learner's second language vocabulary learning.

Lowie $^{[10]}$ believes that "many words in language are related in form, but this relationship is manifested at different levels and varies in intensity". These words that are related in form constitute a family of words. Because they have a common bases or stems. For example, the act word family includes: act, acts, acted, acting, actor, action etc. Words of the same word family are generated by the same word base through derivation or inflection, which greatly reduces the linguistic memory burden of language learners. If the learner remembers the vocabulary through the family of words based on the rules of word formation, they can accumulate a large amount of vocabulary in a relatively short period of time. It goes without saying that second language learners should further enhance the awareness of using word formation rules in the process of vocabulary learning.

\subsection{Using the knowledge of roots and affixes to learn vocabulary}

When learning new words, pay attention to guiding students to learn the roots and morphemes, and let the students analyze and search for the roots of the words themselves. Thereby mastering and expanding the knowledge of roots and affixes. For example, when learning the word extension ${ }^{[11]}$, its word form and morpheme meaning can be decomposed. It consists of the prefix ex-(out) + root tens (stretch, trend) and the -ion noun suffix. The meaning of the word is changed from "stretching out" to "expanding". Then ask the students to think and find other words that contain the same root tens (=stretch), which means "stretch; trend". There are three types of words in the same root with extension.

(1) Adjective: root + adjective suffix -ible,-ile

Tensible (tens "stretching" + ible "can", constituting an adjective, meaning "elongated")

Tensile (tens "tending" + ile "may", constituting an adjective, meaning "extensible ")

Intense (in "strong" +tens "stretch, trend" $+e$, constitute adjective meaning "strong")

(2) Noun: prefix + root + noun suffix -ion, -it

Extension (ex "out" + tens "stretch, trend" + ion, constitute a noun, meaning "expansion ")

Tension (tens "stretch, trend" + ion constitutes a noun, meaning the state of stretching is "tension")

Intensity (in "intensified" + tens "stretch, trend" +ity, constitute noun, meaning "strong, brightness")

(3) Verb: prefix + root + verb suffix -ify, -e

Intensify (adjective intense "enthusiasm" + ify, constitute verb, meaning "enhance")

Tense (tens "stretch, trend" $+e$, constitute verb, meaning "make nervous, make tight")

\subsection{Using morphological knowledge to learn new words}

Words are the carrier of language and culture, and the development of social culture is reflected by the evolution of vocabulary. With the development of society and the advancement of culture, the word has also been updated and evolved into many new words. There are many popular new words in newspapers, online and magazines. Through careful analysis, you can find their part-of-speech features. Such as DINKS (Double Income No Kids), kidult "retaining a childish heart", smize "smile with your eyes", muffin-top "proud flesh at the waist", May-December romance "relationships with significant age differences", Re-tweet depression "when your Weibo is not forwarded, you will feel depressed", $L Q^{[12]}$ "leisure quotient". These words use the above methods of word formation, so it is easy to be understood and remembered by the learner.

\subsection{Analogy and analysis of vocabulary}

Most of the vocabulary of English is structured regularly. This is very helpful for the learner's vocabulary expansion and word memory. In the derivative word, the root is the core part of the word, and the affix is the auxiliary component. When encountering a new word, it is necessary to distinguish which are roots and which are affixes. For example: facile, faculty, factory these three words contain the root of the Latin word fac, fact, fect means to do or to work. The audience, audit, auditor, and auditorium these words contain the Latin root audi, and the audit indicates the concept of "to hear". The four words define, declaim, decrease, and democracy start with de, but they are not the same suffix. Define is consists of the prefix de- "down" + root fin "limit" + suffix - $e$, means "to set the limits down". Declaim is consists of de- "completely" + root claim "to call out", means to call out or to speak loudly, extended to "announce". Decrease is de- "downward" + root creas "to grow" + suffix -e, means to grow downward from a larger amount, extended to "cut back". Democracy is by the root demo "the people" + -cracy noun "power", the meaning of the word is "rule or ruling body by the people", extended to "democratic regime; democratic society".

In the early stages of vocabulary learning, learners first need to go through the so-called project learning phase, which is to memorize words one by one. However, with the introduction of morphological word formation rules, learners can systematically memorize and learn vocabulary. Because English morphological formation rules such as affixation, conversion, compound, and derivative methods have strong productivity. If learners can master and make full use of these rules of morphological formation, their vocabulary learning efficiency will advance by leaps and bounds. In addition, some phenomena discovered by morphologists in the study, such as bracketing paradox, have important implications for language learners ${ }^{[13]}$. Because they can deepen the learner's understanding of the process of the formation of rules such as compound method and derivative method, and provide favorable conditions for them to further deepen vocabulary learning. 


\section{Conclusion}

English has one of the largest vocabularies in the world. Therefore, in order to learn English well, you must master the knowledge of word formation. To acquire English vocabulary comprehensively and in depth, we must rely on scientific theories and methods. In addition, in various forms of examinations, we tend to test students' language ability rather than pure language 3nowledge. At this level, morphological learning can well develop students' language skills and language learning methods. If learners can incorporate these theories and methods into language learning practice, the vocabulary can grow rapidly and lay a solid foundation for the overall improvement of English proficiency.

\section{References}

1. Mccarthy MJ. Vocabulary.Oxford: Oxford University Press; 1990. p. 8.

2.Zhang S, Ren K. Morphology and college English vocabulary teaching (in Chinese). Journal of Southwest University for Nationalities (Humanities and Social Sciences Edition) 2008; 29(S1): 264-266. 3. George Y. Language research. Dai M, He Z (translators). Beijing: Foreign Language Teaching and Research Press; 2000.

4. Hu Z. Course of Linguistics (in Chinese). Beijing: Peking University Press; 1998.

5. Hu A. English vocabulary acquisition from the perspective of morphology (in Chinese). Journal of Jiangxi University of Finance and Economics 2009; 22(6): 141-144.

6. Lu G. Modern English vocabulary (in Chinese). Shanghai: Shanghai Foreign Language Education Press; 1986.

7. Wang S, Wang N. The application of morphology in college English teaching (in Chinese). Journal of Shijiazhuang Vocational and Technical College 2013; 25(04): 69-72.

8. Li P. English roots and vocabulary interpretation of words (in Chinese). Fuzhou: Fujian Education Press; 1984.

9. Li N. A review of researches on knowledge development of metamorphology in English second language (in Chinese). Journal of Southwest University for Nationalities (Humanities and Social Sciences Edition) 2010; 31(S1): 241-243.

10. Lowie WM. The acquisition of interlanguage morphology: A study into the role of morphology in the L2 learner's mental lexicon. Groningen: University of Groningen 1998; 2.

11. Kingsoft Online Dictionary [K/OL]. [2013 Feb 22]. Available from: http://vcom/extension, www.iciba.

12. Yingyudianjing [EB/OL]. [2013 Feb 22]. Available from: http://www.chinadaily.com.cn/language_tips/ trans/ trans_collect. html.

13. Wu Y. The role of morphology in second language learning - Taking English as an example (in Chinese). Journal of Zhejiang Foreign Studies University 2011; (5): 40-45. 